\title{
Utilization of Antibacterial Nanoparticles in Photocurable Additive Manufacturing of Advanced Composites for Improved Public Health
}

\author{
Christopher Billings ${ }^{1}$, Changjie Cai ${ }^{2, *(1)}$ and Yingtao Liu ${ }^{1, *(D)}$ \\ 1 School of Aerospace and Mechanical Engineering, University of Oklahoma, Norman, OK 73019, USA; \\ christopherbillings@ou.edu \\ 2 Department of Occupational and Environmental Health, University of Oklahoma Health Sciences Center, \\ University of Oklahoma, Oklahoma City, OK 73104, USA \\ * Correspondence: changjie-cai@ouhsc.edu (C.C.); yingtao@ou.edu (Y.L.); \\ Tel.: +1-405-271-8001 (ext. 46774) (C.C.); +1-405-325-3663 (Y.L.)
}

Citation: Billings, C.; Cai, C.; Liu, Y. Utilization of Antibacterial Nanoparticles in Photocurable Additive Manufacturing of Advanced Composites for Improved Public Health. Polymers 2021, 13, 2616. https://doi.org/10.3390/ polym 13162616

Academic Editor: Rafiq Ahmad

Received: 17 July 2021

Accepted: 5 August 2021

Published: 6 August 2021

Publisher's Note: MDPI stays neutral with regard to jurisdictional claims in published maps and institutional affiliations.

Copyright: (c) 2021 by the authors. Licensee MDPI, Basel, Switzerland. This article is an open access article distributed under the terms and conditions of the Creative Commons Attribution (CC BY) license (https:/ / creativecommons.org/licenses/by/ $4.0 /)$.

\begin{abstract}
This paper presents the additive manufacturing and characterization of nanoparticlereinforced photocurable resin-based nanocomposites with a potential antimicrobial function for improved public health applications. Two types of photocurable resins are reinforced by titanium dioxide $\left(\mathrm{TiO}_{2}\right)$ or zinc oxide $(\mathrm{ZnO})$ nanoparticles with average diameters in the 10-30 nm range to provide antimicrobial properties. The developed nanocomposites can be additively manufactured using the digital light processing method with an outstanding surface quality and precise geometrical accuracy. Experimental characterizations are conducted to investigate key mechanical properties of the 3D printed nanocomposites, including Young's Modulus, tensile strength, and abrasion resistance. Specimens produced were observed to demonstrate the following characteristics during testing. Tensile strength increased by $42.2 \%$ at a maximum value of $29.53 \mathrm{MPa}$. The modulus of elasticity increased by $14.3 \%$, and abrasion resistance increased by $15.8 \%$. The proper dispersion of the nanoparticles within the cured resin is validated by scanning electron images. The wettability and water absorption testing results indicate that the developed nanocomposites have an outstanding water resistance capability. The pairing of digital light processing with these novel nanocomposites allows for the creation of complex composite geometries that are not capable through other manufacturing processes. Therefore, they have the potential for long-term usage to improve general public health with antimicrobial functionality. The pairing of an unmodified photocurable resin with a $1 \% \mathrm{ZnO}$ concentration demonstrated the most promise for commercial applications.
\end{abstract}

Keywords: additive manufacturing; photopolymerization; digital light processing; nanoparticles; antimicrobial; public health

\section{Introduction}

Additive manufacturing (AM) has played a significant role in polymer and composite manufacturing for broad biomedical and public health applications in recent years [? ? ? ]. Due to the broad array of AM techniques, such as fused deposition modeling and direct ink writing, traditional biocompatible polymers and composites can be rapidly converted into novel devices and products to enhance medical treatment and public health [? ]. Additionally, the integration of AM technologies with novel polymers and nanomaterials with beneficial functionalities has opened new directions for developing cuttingedge devices, sensors, and tools that may revolutionize medical treatment and prevent infectious diseases.

Photocurable-based AM is a unique manufacturing option due to the high product quality and low processing temperature. These types of AM systems are usually referred to as stereolithography (SLA) and digital light processing (DLP), and use an ultraviolet 
light source to cure photopolymers layer by layer with a high spatial resolution and surface quality [? ? ]. This feature allows the photopolymerization-based AM systems to create parts with almost perfect microstructures, which is rare for other AM processes, such as fused deposition modeling. The build plate is the only moving part in these machines traveling in the $\mathrm{Z}$ direction. This mechanically simple design allows for a precise process control and optimization during AM for various photocurable polymers and nanoparticle-reinforced composites.

Wear resistance, durability, and water resistance of polymers and nanocomposites are critical in healthcare environment applications. For example, Clostridioides difficile (C. difficile) infection (CDI) is the leading cause of nosocomial diarrhea worldwide with substantial morbidity, mortality, and healthcare cost [? ? ]. In addition, the Centers for Disease Control and Prevention (CDC) has classified C. difficile as an "urgent" threat (the highest threat) in its antimicrobial resistance threat report [? ]. Contact precautions and environmental hygiene have been only moderately successful for CDI control, which remains a seemingly intractable problem. The challenging is that the $C$. difficile spores that become airborne during patient care activities travel long distances, contaminating environmental surfaces remote from the sources [? ]. Thus, CDI is related to many healthcare activities, including the bed making, bedpan washing, provider movement, housekeeping activities, and toilet flushing [? ? ? ]. The $C$. difficile bacteria can grow and be emitted from both wet (such as flushing toilet bowl water and bowl surfaces) and dry (such as table, ground, and wall surfaces) environments. Therefore, it is critical to test the key mechanical properties of the manufactured composites to prevent the bacteria growth and, then, to reduce their emissions.

Nanoparticles (particle diameter $<100 \mathrm{~nm}$ ), such as titanium dioxide $\left(\mathrm{TiO}_{2}\right)$ and zinc oxide $(\mathrm{ZnO})$, have been widely studied due to their significant antimicrobial potentials, especially valuable to antibiotic-resistant bacteria [? ? ]. For example, when Escherichia coli (E. coli) colonies were exposed to surfaces treated with nanoparticles, prevention in further bacteria growth was observed [? ]. The nanoparticle antimicrobial capacities can be further enhanced by ultraviolet (UV) illumination due to the photocatalysis effects of these nanoparticles [? ? ]. UV illumination was observed to be a vital mechanism in activating the antibacterial properties of the given nanoparticles [? ? ? ? ]. The size-controlled nanoparticles can also improve the antibacterial efficacy because the smaller sizes can easily penetrate into bacterial membranes due to their larger particle surface areas [? ? ]. If exposed to light in the UV spectrum, the nanoparticles became safe for use in the human body.

Testing of these nanoparticles relies on the test surface having a light dispersion of the nanoparticle to work. To achieve these traits in a polymer application, it would be required for the part to have a continuous surface coating of the nanoparticle on all surfaces. This coating would have to either be wear-resistant or rely on a mechanism that ensures new nanoparticles are exposed to the surface during wear. In addition, proper particle dispersion would have to be investigated through a scanning electron microscope (SEM). Once the part's surface is successfully coated with the nanoparticle, antibacterial traits can be expected to be seen.

A solution to the above issue is to create a nanocomposite polymer. This nanocomposite would have to show a proper dispersion throughout the entirety of the part and have a large enough concentration of the selected nanoparticle to be effective. A strong candidate for the matrix material would be a photocurable resin due to the material's workability before activation. Ensuring the viscosity of the matrix is low enough that the addition of the nanoparticle will not hinder use case performance is less of a concern when using liquid resins [? ]. Using proper mixing methods and quick curing will allow for a homogenous nanocomposite that would not only show nanoparticles on the surface but would continuously expose new nanoparticles as the material degrades over time.

Using photocurable-based AM processes with a nanocomposite resin as suggested above, would allow for a complex model creation, high-speed production, and antibacterial 
traits. Utilizing LCD-based curing technology would allow for entire layers to be cured simultaneously to ensure the chemical reaction of the curing polymer would not disturb the surrounding nanocomposite mixture. Nanoparticles such as $\mathrm{TiO}_{2}$ have been successfully used in AM techniques to manufacture nanocomposite structures [? ]. The inclusion of $\mathrm{ZnO}$ will allow for a direct comparison of two different nanoparticles that both inhibit bacterial growth and their effect on the AM process.

This research addresses the high cost and complexity associated with producing composites using traditional methods by creating a novel antibacterial nanocomposite designed for use in digital light processing machines. Utilizing two different proven antibacterial nanoparticles and two different matrix materials, researchers explore the physical properties of the novel nanocomposites and their effect on the manufacturing process. These nanoparticles are chosen due to their already current use in nanofilms and tools found in the healthcare industry [? ? ? ]. These nanocomposites paired with AM allow for creating specialty parts and tools vital to the healthcare industry.

\section{Materials and Methods}

\subsection{Equipment Used}

The DLP-based polymer 3D printer used to conduct this study was an Anycubic Photon (Anycubic, Commerce, CA, USA) with a build volume of $115 \mathrm{~mm} \times 65 \mathrm{~mm} \times 155 \mathrm{~mm}$. This printer has a $y$-axis resolution of $1.25 \mu$ and a $z$-axis resolution of $25 \mu$. The printer utilizes an LCD panel to mask off LEDs that emit light in the $405 \mathrm{~nm}$ wavelength. No physical modifications to the printer were performed. The two resins that were used were produced by ELEGOO Mars (Elegoo, Shenzhen, China) and were a standard LCD UV-curing photopolymer rapid resin and an ABS-like LCD UV-curing photopolymer rapid resin. The two nanoparticles selected were $\mathrm{TiO}_{2}$ and $\mathrm{ZnO}$ in the $10-30 \mathrm{~nm}$ range from Sky Spring Nanomaterials (Sky Spring, Houston, TX, USA). For tensile testing, an Instron 5969 (Instron, Norwood, MA, USA) was used with a $5 \mathrm{kN}$ load cell. Abrasion tests were completed using a LaboPol-5 (Struers Cleveland, OH, USA) with a LaboForce-3 head attachment. A grinding disc with aluminum oxide abrasive sized at $50 \mu$, or 240 grit pad, was used as the grinding medium.

\subsection{Nanocomposite Synthesis Requirements}

To synthesize the nanocomposite resin, several steps were followed to help increase printability. Initially, the ability for the nanocomposite to cure required testing as the addition of a non-curable agent led to reduced bonding and light transmission throughout the print. The software used for preparing prints, CHITUBOX, allowed for modification of all printing steps. The criteria used to determine successful prints included the ability for the print to reliably adhere to the build plate and for the printer to have a minimal loss in part resolution. The addition of the nanoparticle to the resin created two issues. The first issue was that as the nanoparticle percentage increased, the viscosity of the novel composite also increased. This directly affected the printer's ability to reset between layers and allowed for an even nanoparticle dispersion. The second issue was that as the nanoparticle percentage increased, the ability for the printer to cure each layer adequately decreased. The modified parameters were the number of initial layers, the initial layer cure time, the remaining layer cure time, and the initial $\mathrm{z}$ distance. The first issue was addressed using different concentrations of nanoparticles to test for proper return flow into the recess created due to the curing part. As the printer finished a layer, the build plate rose above, releasing the part from the FEP sheet to allow the new resin to flow into the recess. The time could be adjusted through software but was left constant to keep print times at a reasonable speed. Increasing this time would also create issues with particle settlement. Results showed that concentrations up to $5 \%$ could adequately fill the void in the time provided. To address the second issue of reducing bonding between the resin and the build plate, the initial $\mathrm{z}$ height was decreased to create a thinner layer of composite between the FEP sheet and the aluminum build plate. This helped ensure that the resin 
was forced into the micro-abrasions on the build plate, therefore, increasing build plate adhesion. This also reduced the amount of resin that required curing on the first layer.

Along with this change, the initial layer curing time was doubled from $60 \mathrm{~s}$ to $120 \mathrm{~s}$. An increased cure time would help improve the consistency of the cure across the layers. A total of 5 bottom layers were used for the prints. Lastly, the cure time for the remaining layers was increased from $8 \mathrm{~s}$ to $15 \mathrm{~s}$. With these settings in place, varying concentrations of nanoparticles were added to the composite for testing. Adequate bonding and detail retention were easily held at a $1 \%$ nanoparticle addition. At around $2.5 \%$ addition, failed prints began to arise due to a failure in the bonding of the first layer.

There were several different mixing methods employed to introduce the nanoparticles to the matrix resins. First was a process of hand mixing the two components for five minutes with a stir rod. The second was to shake the mixture in a closed bottle until no visible particles could be identified. The last method was to use a stirring plate. Hand mixing was an effective procedure but required careful inspection of the mixture as it is poured into the printer to ensure the mixture is homogenous without the formation of clumps. Filters with pore diameters of less than $1 \mathrm{~mm}$ could be employed to help ensure no large particles were introduced into the printing vat, leading to print failures. The second method provided a greater degree of homogeneity throughout the mixture due to the forces involved in the mixing process and the ability to change the direction of the fluid flow within the mixing chamber. The last method provided similar results to the first as the stirring plate would often have difficulty mixing the resin at speeds above $100 \mathrm{rpm}$ due to the high viscosity of the mixture. The resins mixed in the enclosed bottle also showed less particle settlement. A proper mixture would suspend particles for up to $6 \mathrm{~h}$ of printing without needing to be remixed. Therefore, an enclosed centrifugal mixer is recommended as the best machine for synthesizing the nanocomposite resin. The entire manufacturing process for the resin and part is depicted in Figure ??.

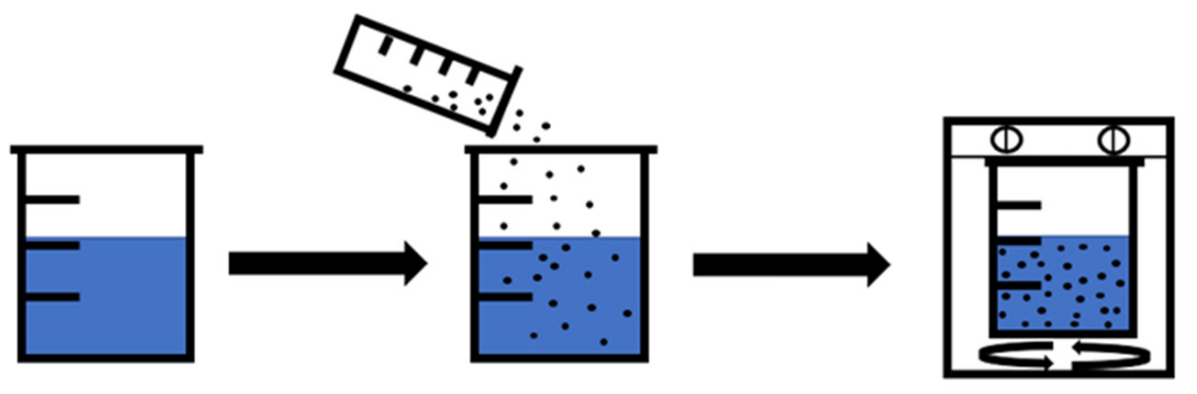

Virgin Resin Material

Add in Nanoparticle

Mix materials for 5 $\min$

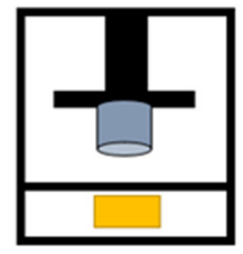

Print Desired Part With Modified Curing Times

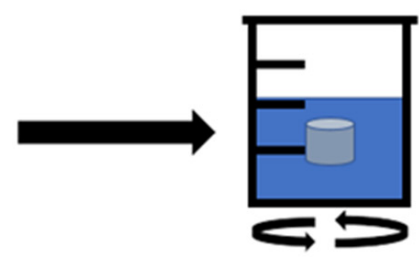

Wash Part In Isopropanol Alcohol

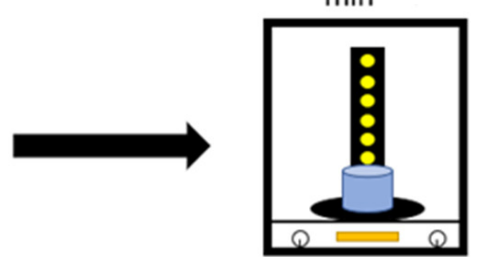

Cure Part For End Use

Figure 1. Complete manufacturing process.

\subsection{Addressing Particle Settlement}

Other essential concerns with the use of a non-homogenous nanocomposite included particle settlement over time. As the only motion during the print was the build plate repeatedly raising and lowering, the nanoparticles would begin to settle over time as their density was higher than that of the resin matrix. This issue was most noticeable at higher particle concentrations and would cause layer adhesion issues during the print as the 
particles settled and increased the relative concentration at that layer. There were several approaches used to remedy this issue. The first approach was to start with a minimal amount of nanoparticle addition, as previously discussed, as this would help ensure proper distribution throughout the matrix material. The second and more proactive step was to pause the print on the hour and remix the composite by hand. This was performed in the printer with a plastic scraper as not to damage the FEP sheet. Finally, removing the resin vat could cause alignment issues with the printer and, therefore, the mixing was performed inside the vat with the build plate raised to the maximum $\mathrm{Z}$ height.

\subsection{Post Processing}

After removal from the build plate, all parts were put into an isopropyl alcohol bath on a stirring plate for a minimum of 6 minutes. This allowed for the isopropyl alcohol to dissolve any remaining uncured resin on the parts, which is critical to retaining high part resolution during the final stage. After a thorough cleaning, the part was put into a UV chamber for a minimum of $4 \mathrm{~min}$. Again, time was added based on the appearance of the part until a flat matte sheen was achieved.

\subsection{Part Selection}

To demonstrate the 3D printability of the novel nanocomposite, several different complex parts were printed and assembled. The first of the three primary tests that were conducted consisted of a chess rook with intricate detail to observe the nanocomposite resin's ability to maintain surface details and internal geometries within a single part. Secondly, a large pipe fitting was printed to show the material's ability to be used in fluid flow systems successfully. Lastly, a prosthetic finger was printed to give a direct example of a biomedical application. All these parts can be seen in Figure ??a. Along with these prints, over 30 tensile test specimens were printed.

(a)

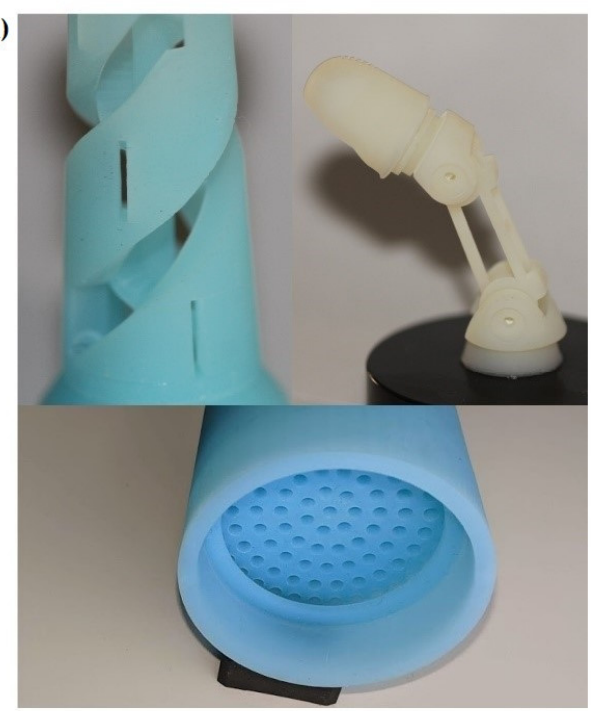

(b)

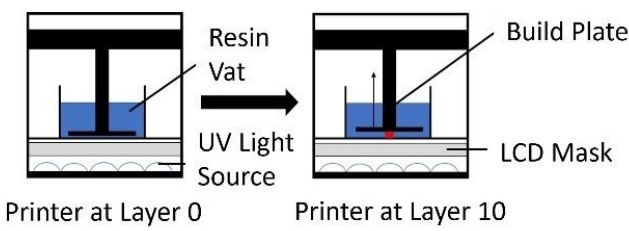

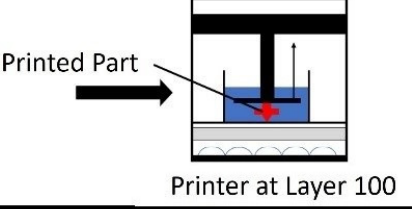

(c)

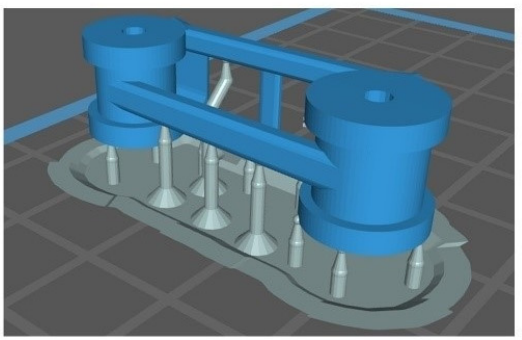

Figure 2. (a) Three-dimensional printed example pieces included a chess rook, a prosthetic finger, and a pipe fitting. (b) The manufacturing process for LCD-based printing systems. (c) Middle finger joint in blue and support material in grey.

The pipe fitting was printed to showcase the part's ability to be used in a fluid flow system. As discussed later, the low water absorption of these novel resins over time allowed for part adaptation into fluid environments where antibacterial properties were beneficial. Many parts of the healthcare industry are often in contact with different types of fluids and must be meticulously cleaned as a moist environment breeds bacteria. The fittings 
successfully printed here showcased the ability to produce complex part geometries that would help lead to less turbulent flow within a system. This would be incredibly beneficial when working with fluid systems where turbulent flow that traps air bubbles is undesirable. Additionally, the nature of the material would lead to fewer concerns for contamination and longer implementation cycles before cleaning or replacement. As these parts can be produced in mere hours for a fraction of the cost of most medical equipment, the viability of this technology in medical environments increases.

Lastly, a working prosthetic finger designed by Danger Creations was printed to show the opportunities this technology provides. The part has many intricate details and moving parts that perfectly interface together. The prosthetic finger can be manufactured in under $2 \mathrm{~h}$. This technology allows for weight optimization of medical apparatuses that are not possible with other manufacturing techniques. Since weight is a critical factor in user experience and comfort, customizable options such as these are consumeroriented. Because the novel nanocomposite was developed explicitly for use in digital light processing machines, the design freedom given to medical engineers was greatly expanded. In addition, the material cost for this finger was just 18 cents for the nanoparticles and resin material. Given a uniform dispersion, a low concentration of nanoparticles led to a low-cost nanocomposite. The pairing of a low-cost nanocomposite with AM technology provides the best solution for corporations needing intricate one-off parts. Due to the elimination of expensive machinery and custom molding, this technology offers an unparalleled cost advantage for the healthcare industry. This drastic reduction in cost and manufacturing time will revolutionize the medical industry if implemented on a per hospital level, all while delivering better care.

\subsection{Tensile Testing}

Tensile testing was performed using the ASTM bar-type IV standard to verify uniformity between prints and the effect of the nanoparticle on the physical characteristics of the base resin materials. The specimens were $70 \mathrm{~mm}$ in length as this maximized the build plate area that was available. In addition, this was performed so that it would show up as lower tensile stress values if there were nonuniformity throughout the material.

\section{Results}

\subsection{Nanoparticle Dispersion Methods}

Extensive testing was performed to produce complex geometries and parts that could not be traditionally manufactured using subtractive or casting methods. Within this scope, special attention was given to the printer's ability to hold tolerances and detail resolution throughout the entirety of the print. As this technology could be implemented in the biomedical field, high-speed fabrication and low failure rates are critical to widespread adoption.

\subsubsection{Comparing the Matrix Materials}

The first dataset analyzed was the difference in tensile strength of the ABS-like resin and the control resin. Testing showed that both materials performed similarly, with the base resin just edging out the ABS-like material in terms of tensile stress by $1.42 \mathrm{MPa}$. This equates to just a $6.8 \%$ increase in tensile strength for the base resin. As far as strain, both materials showed nearly identical strain rates at 0.075 . The main difference between the two resins was that the standard deviation for the ABS-like photopolymer was lower at a value of $1.636 \mathrm{MPa}$ compared to the base resin standard deviation of $2.856 \mathrm{MPa}$. This increase in deviation was likely the cause for the slightly higher average tensile stress seen in the resin samples.

\subsubsection{Analysis of Base Resin Nanocomposites}

The 3D printed nanocomposites with $1 \mathrm{wt} \%$ nanoparticles demonstrated a significant increase in tensile strength and Young's Modulus over the control. Both the $\mathrm{ZnO}$ and $\mathrm{TiO}_{2}$ 
had a positive effect on the material's physical tensile strength. The $\mathrm{ZnO}$ led to an average tensile stress of $33.696 \mathrm{MPa}$, and the $\mathrm{TiO}_{2}$ led to an average tensile stress of $29.533 \mathrm{MPa}$. Along with this, the average strain reduced significantly in both samples. Up to an $88 \%$ increase in modulus was observed for the $\mathrm{ZnO}$ specimens as depicted in Figure ?? The $\mathrm{ZnO}$ sample was reduced to a strain rate of 0.036 and the $\mathrm{TiO}_{2}$ sample reduced to 0.026 . Thus, the base resin material was a strong candidate for a nanocomposite matrix as the tensile strength increased by $42.2 \%$ and the strain reduced by at least $50 \%$.
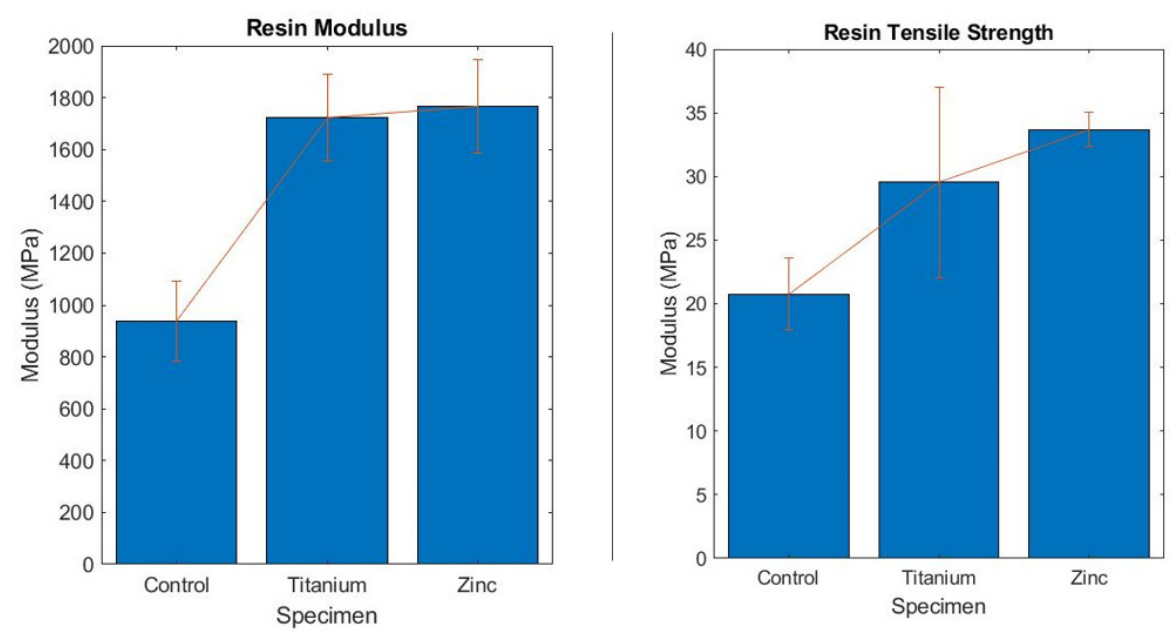

Figure 3. Young's modulus and ultimate tensile strength bar graphs comparing the resin control and nanocomposites.

\subsubsection{Analysis of ABS Nanocomposites}

The ABS-like resin showed a negative correlation with the addition of nanoparticles. Both the $\mathrm{ZnO}$ and $\mathrm{TiO}_{2}$ decreased the tensile strength of the specimens as depicted in Figure ??. The $\mathrm{ZnO}$ led to a $15.1 \%$ decrease in tensile strength, while the $\mathrm{TiO}_{2}$ led to a $12.98 \%$ decrease. The modulus reduced by $1.7 \%$ for the titanium samples and $4.4 \%$ for the zinc. The strain reduction was only $41 \%$ for the $\mathrm{ZnO}$ and $42 \%$ for the $\mathrm{TiO}_{2}$. One improvement of the novel nanocomposite was reducing the standard deviation of between 1.03 MPa and 0.81 MPa compared to the ABS-like resin. The reduction in the Young's modulus and tensile strength in the ABS resin could have been caused by the ununiform dispersion of nanoparticles. Since the ABS resin had an increased viscosity, the dispersion difficulty of nanoparticles in ABS increased dramatically. An improved nanoparticle dispersion can lead to improved mechanical properties in novel nanocomposites.
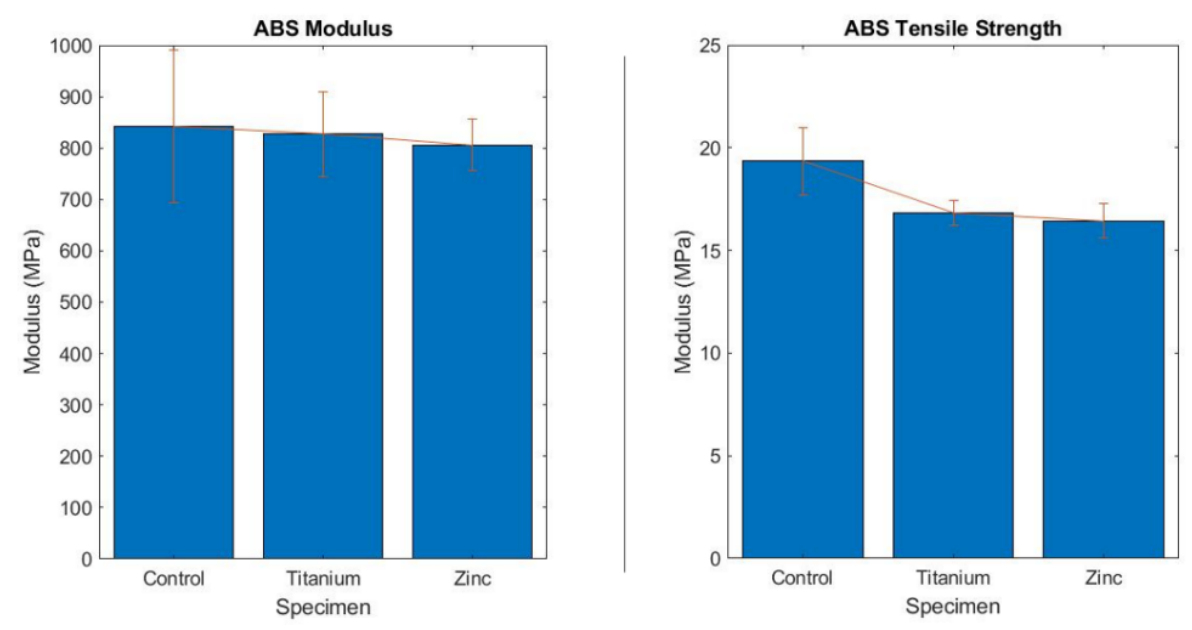

Figure 4. Young's modulus and ultimate tensile strength bar graphs comparing the ABS control and nanocomposites. 


\subsection{Abrasion Testing}

The abrasion testing was conducted under a constant water stream and at a pressure of $15.9 \mathrm{KPa}$. The grinding disc was aluminum oxide with 50-micron-sized abrasives. The disc was rotated at a speed of $100 \mathrm{rpm}$ and the head that held the sample was rotated at $250 \mathrm{rpm}$. Each sample was put through the grinding process for $5 \mathrm{~min}$ at $30 \mathrm{~s}$ intervals. The procedure is depicted in Figure ??a.

(a)

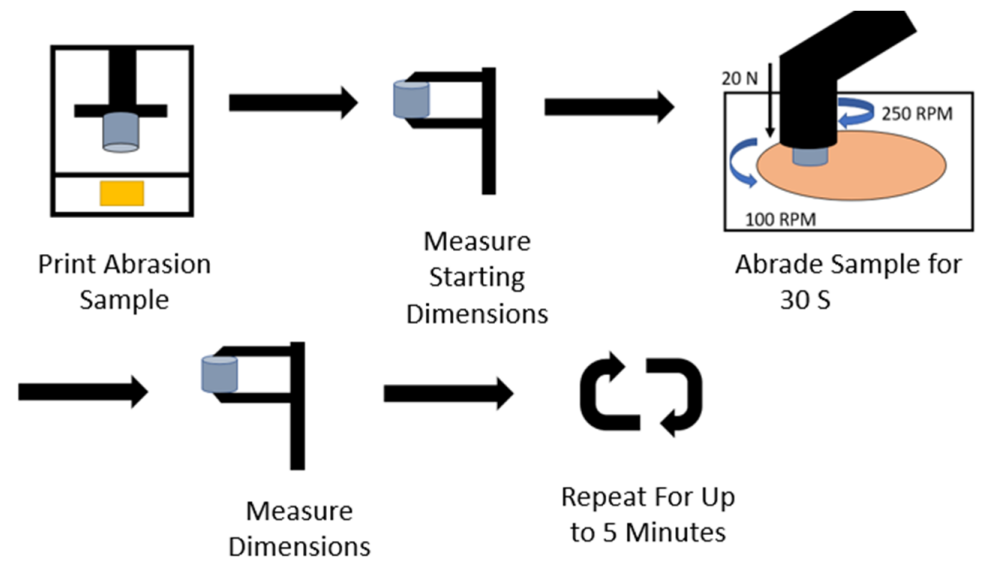

(b)

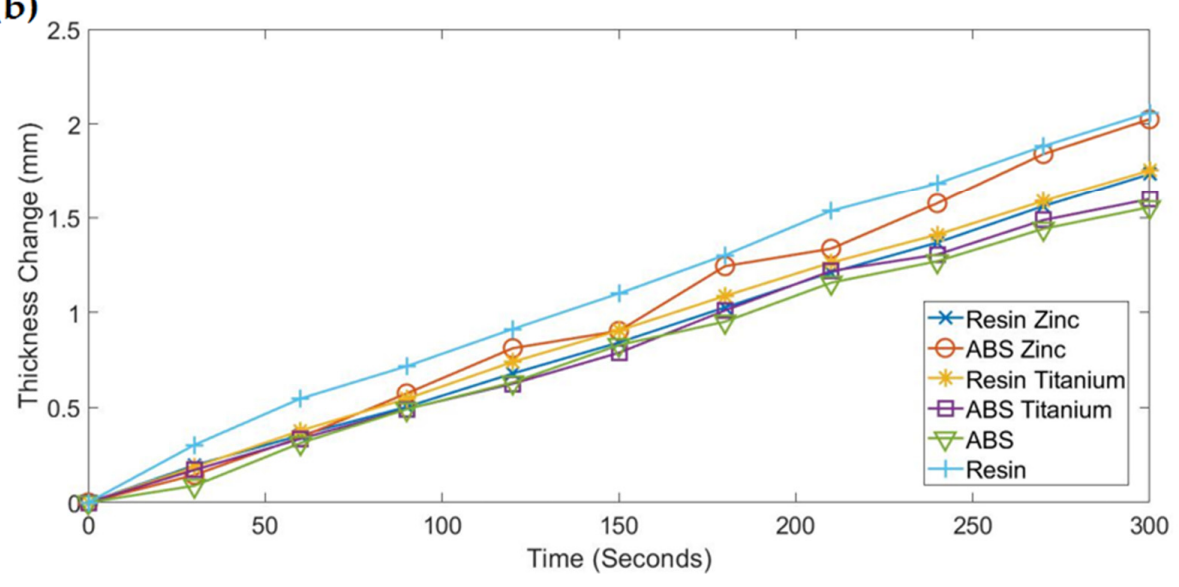

Figure 5. (a) Abrasion testing procedure for all samples. (b) Abrasion testing results compared across 5 min of abrasion.

Starting with the control resins, the ABS-like resin had the least material degradation of all samples at just $1.55 \mathrm{~mm}$. Comparatively, the non-ABS resin had the highest amount of material degradation at $2.059 \mathrm{~mm}$. These tests are equivalent to dragging a part over $471 \mathrm{~m}$ of abrasive material with $15.9 \mathrm{KPa}$ of pressure. This equated to over $300 \mathrm{~m}$ of abrasion per millimeter of thickness change for the ABS-like photopolymer and over $225 \mathrm{~m}$ for the resin.

The nanocomposite samples fell between the above two base samples, with the most notable distinction being the added abrasion resistance in the resin samples. Both resin nanocomposites performed very similarly with a thickness reduction of $1.733 \mathrm{~mm}$ for the $\mathrm{ZnO}$ resin nanocomposite and $1.752 \mathrm{~mm}$ for the $\mathrm{TiO}_{2}$ resin nanocomposite. These samples showed a $15.8 \%$ increase in abrasion resistance compared to the non-modified material. In contrast, both ABS-like resin nanocomposites showed a decrease in abrasion resistance. The ABS $\mathrm{ZnO}$ nanocomposite performed second worst with a thickness change of $2.024 \mathrm{~mm}$. The $\mathrm{ABS} \mathrm{TiO}_{2}$ sample showed a degradation of $1.598 \mathrm{~mm}$, which was still slightly better than all other non-ABS tests.

\subsection{Water Contact Angles and Water Absorption}

The last two physical tests performed were water contact angle tests and water absorption tests. These tests were performed to characterize the material's ability to 
be used in wet environments for extended periods. The water contact angle measurements were completed for all six specimens using ImageJ software's drop snake analysis [? ]. The factory resin showed an average contact angle of 68.8 degrees. All water contact angle tests were performed on the printed surface with no alterations performed post-printing. The $\mathrm{TiO}_{2}$ was observed to reduce the water contact angle to an average of 52.85 degrees. This reduction was also seen in the $\mathrm{ZnO}$ nanocomposite but to a lesser degree, achieving an average contact angle of 60.85 . As shown in Figure ??, the titanium nanocomposite had the lowest water contact angle across all tests for the resin matrix.
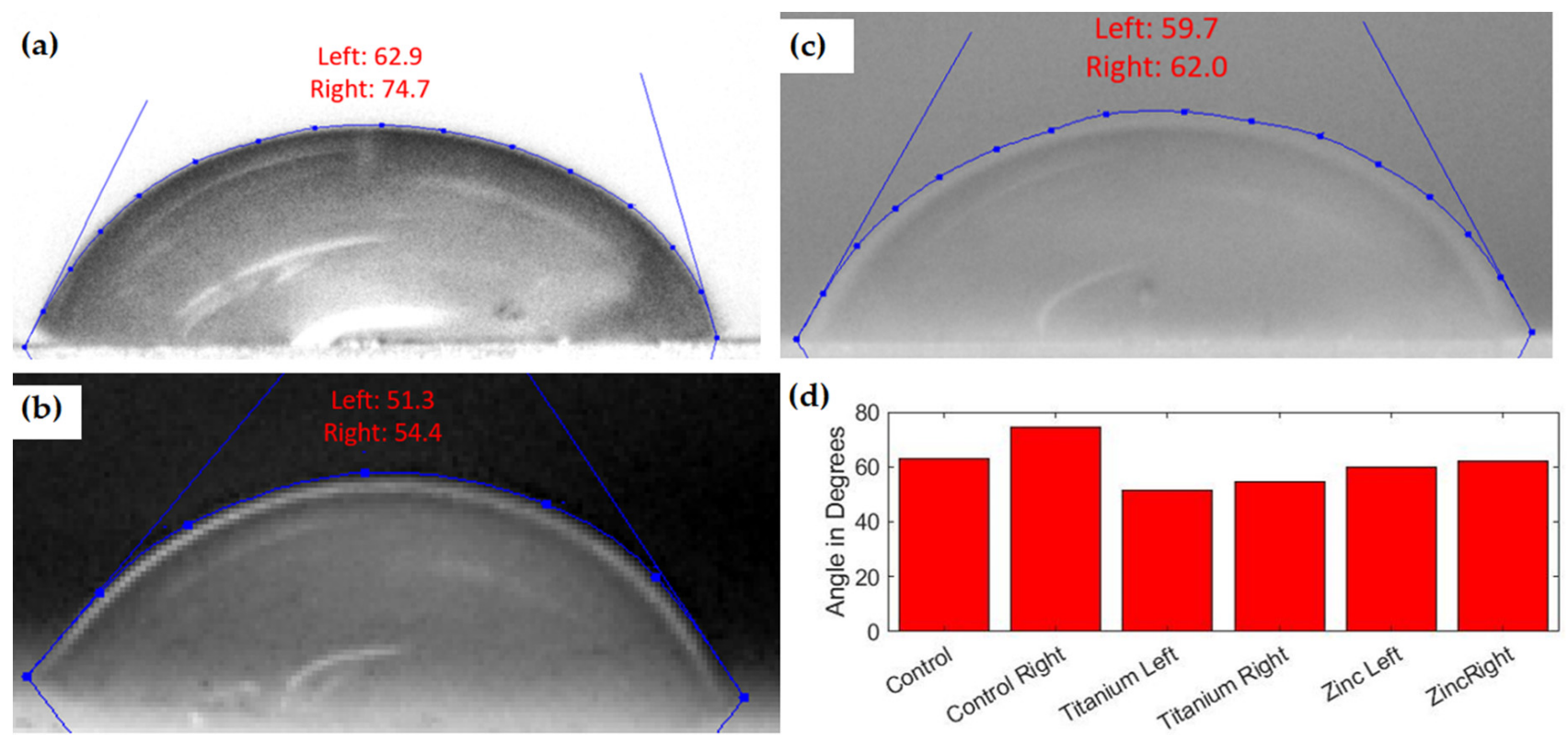

Figure 6. (a) Control resin water contact angles. (b) $\mathrm{TiO}_{2}$ resin nanocomposite water contact angles. (c) $\mathrm{ZnO}$ resin nanocomposite water contact angles. (d) Bar graph of all resin water contact angles.

The ABS matrix performed similarly but with higher overall values for all tests. The control contact angle was $16.1 \%$ higher at an average of 79.9 degrees. This larger contact angle was observed to affect nanocomposites similarly with average contact angles of 60.5 and 71.7 degrees for $\mathrm{TiO}_{2}$ and $\mathrm{ZnO}$ nanocomposites, respectively. As observed in the resin specimens, the $\mathrm{TiO}_{2}$ also demonstrated the lowest water contact angle overall for the $\mathrm{ABS}$ matrix material, with all data observed shown in Figure ??.

Since the parts demonstrate hydrophobic properties, it was vital to ensure that the water absorption of the material was relatively low to ensure that they could still be applicable in wet environments. Water absorption testing performed followed ASTM D570 for plastics. The tests were performed for over 20 days and the maximum water absorption rate recorded was $4 \%$ by weight-the absorption rate plateaued at day 15 following a logarithmic curve as shown in Figure ??. Within the first $24 \mathrm{~h}$, the samples saw a $1.39 \%$ gain in weight and a $1.73 \%$ gain over the first seven days. The specimens were checked after six months of continued submersion and saw no increase past the $4 \%$ observed in the first 15 days. Specimens were left at room temperature for all tests. 
(a)

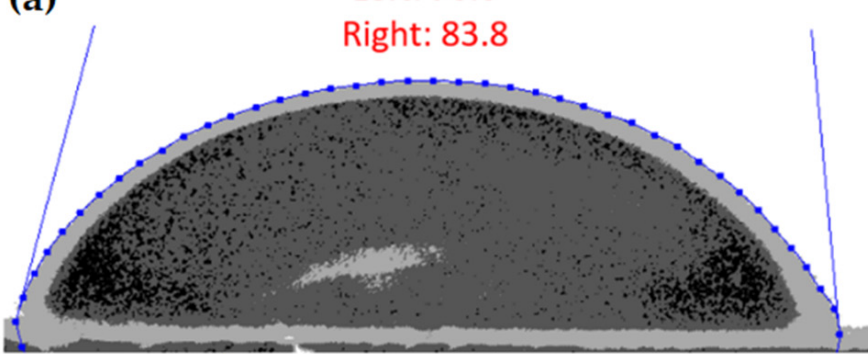

\section{(b)}

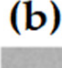

Left: 76.0

Left: 63.3

Right: 57.4

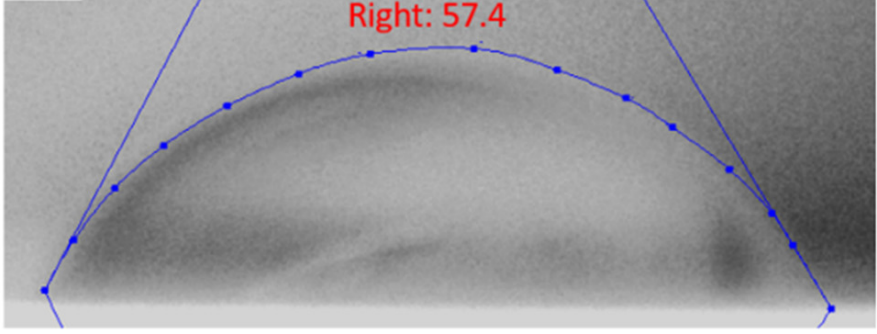

(c)
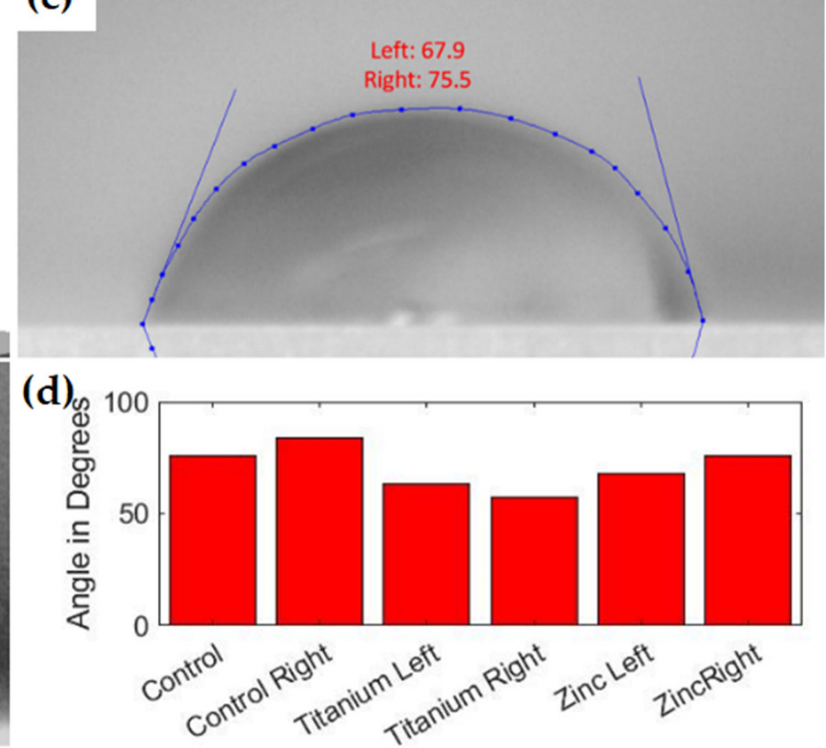

Figure 7. (a) Control ABS water contact angles. (b) $\mathrm{TiO}_{2}$ ABS nanocomposite water contact angles. (c) ZnO ABS nanocomposite water contact angles. (d) Bar graph of all ABS water contact angles.

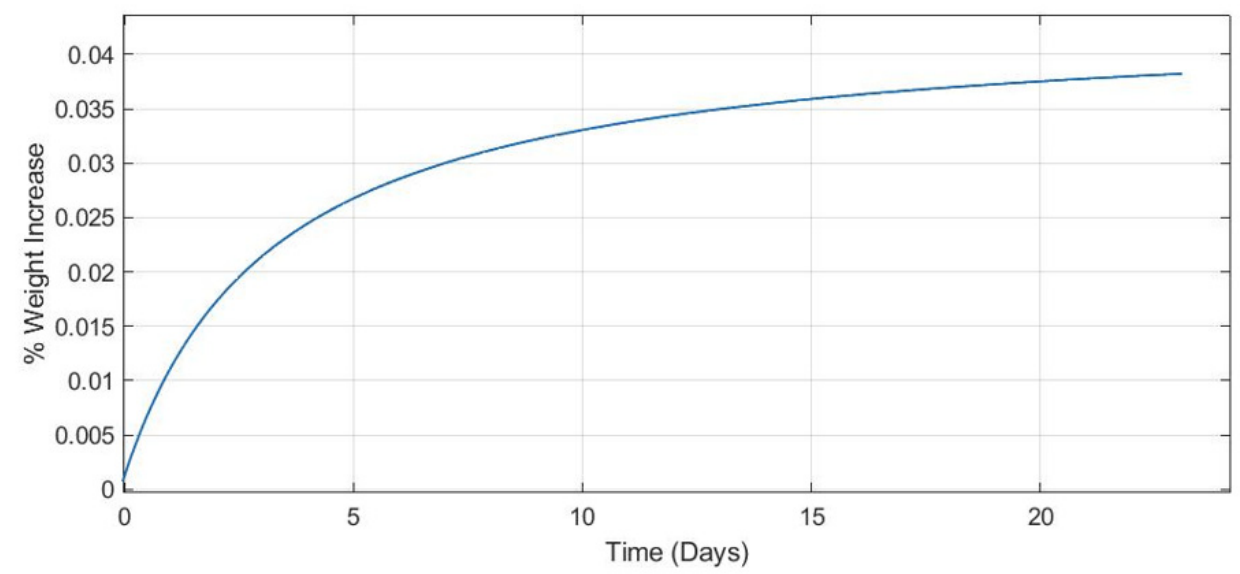

Figure 8. Water absorption percentage over time.

\subsection{SEM Analysis}

SEM provided a validation of the homogenous dispersion across the surface of the printed pieces. An even dispersion with little clumping was required to observe antibacterial traits on the surface of the prints. The SEM images provided in Figure ?? show $\mathrm{TiO}_{2}$ and $\mathrm{ZnO}$ on the surface of the resin matrix. Both images were taken from parts produced on the printer with no modification except for sputter coating, which was required for the SEM process. The nanoparticles can be seen circled in red on each of the below images. Again, minimal clumping was observed, and the dispersion was excellent across the entire inspected image. 

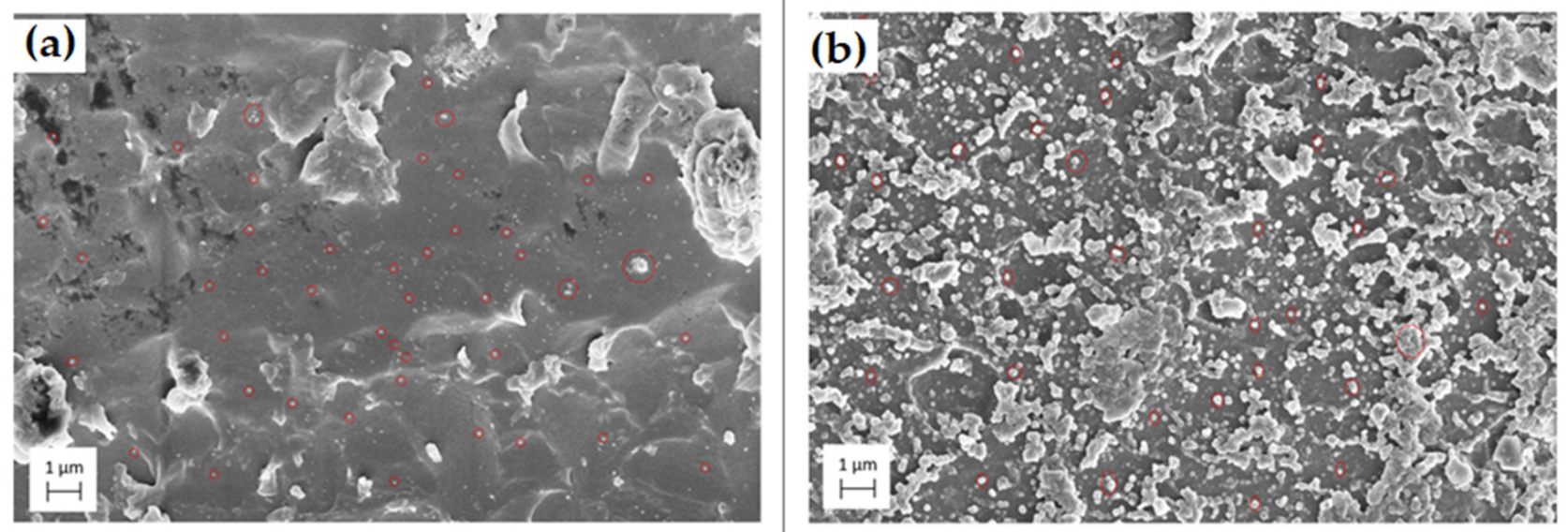

Figure 9. (a) SEM imagery of $\mathrm{TiO}_{2}$ particles in a resin sample. (b) SEM imagery of $\mathrm{ZnO}$ particles in a resin sample.

\section{Discussion}

\subsection{Three-Dimensional Printing Applications}

The critical discovery within this work was the rapid synthesis and production of antibacterial parts using 3D printing technologies. The ability for non-specialists to produce custom parts in under several hours specifically designed for the medical field will help speed up the adoption of 3D printing into the medical sector. The materials used were easy to handle and store while only requiring basic safety measures such as nitrile gloves and eye protection. Therefore, hospitals will not require specialized training and facilities for implementation. Photocurable-based AM has already proven to be one of the best-suited technologies for the healthcare industry [? ? ? ? ]. LCD-based printing systems also offer a significant advantage in processing speed as entire layers can be cured at once compared to more expensive SLA machines [? ]. The most notable example was the prosthetic finger, as all pieces were printed on one build plate.

Due to the high accuracy possible with these machines, it is straightforward to manufacture parts that interface together [? ]. The prosthetic finger example can be assembled in mere min and can easily be sized to any individual. The design freedoms available when using AM are widely known, but implementing these machines into end-use cases has been problematic due to the array of downsides associated with this new technology. Photocurable 3D printing paired with novel nanocomposites, as documented here, suffers from very little of the disadvantages that other 3D printing technologies suffer from. The part's physical properties are entirely suitable for biomedical uses, the accuracy and resolution are well within desired tolerances, and the ease of manufacturing allows for trouble-free manufacturing.

Resins' unique printing ability allows for a part to be printed with no visible layer lines. A design of a 3D chess rook was printed in an ABS ZnO nanocomposite to showcase the intricate detail that this novel resin can uphold while still being fully functional. As the printing process is performed layer by layer, intricate details such as internal features can be produced, as seen by the internal staircase on the rook. The other benefit is that large hollow cavities can be drained of resin during the washing process and cured empty to reduce the overall weight. This leads to design freedom not abundantly found in composite manufacturing and, most importantly, not easily implemented in traditional manufacturing.

\subsection{Physical Properties}

Tensile testing results have shown two interesting critical differences between the novel nanocomposites. The first noticeable data point was the similarities between the 
factory resin materials when looking solely at tensile strength. The average tensile strength between the two materials differed by only $7 \%$. Along with this, the strain rate differed by only $0.27 \%$. The standard deviation for the resin was noticeably higher at a value of 2.856 , leading to a $95 \%$ statistical confidence interval that both sample's mean tensile stress values were within the same range. These data appeared to show that both factory materials were physically no different when a tensile force was applied.

Analyzing the abrasion data was where the difference between the two materials became apparent. The factory ABS-like photopolymer outperformed all other tested materials and had a $24.7 \%$ increase in abrasion resistance compared to the factory resin. This difference constitutes the main design point behind the ABS-like material when printing end-use functional parts. This increase in abrasion resistance also makes the material a strong candidate for implementation where low stresses will be seen, but high traffic is expected.

The addition of the nanoparticles to both matrix resin materials was where the data started to differ drastically. The nanocomposite addition reduced the properties of the ABSlike photopolymer in every test performed, whereas it improved the properties in every test for the resin material. The only exception to this was the water contact angle. As seen in the tensile test Figure ??, the lower physical properties were not caused by anomalies during the printing process as each part failed at similar yield strengths. In addition, the lowest standard deviation of all sample tests was in both ABS nanocomposite resins. This leads to the inference that the ABS-like resin's chemical makeup was not a strong candidate for nanoparticle addition. The probable cause of this is that during the curing process, the adhesion of the ABS-like resin and the nanoparticle was less than ideal. The lower results could be a side effect of micro-fractures caused by an incomplete bond between the nanoparticle and matrix. The only test where the addition of the nanoparticle had a minimal effect was within the ZnO ABS nanocomposite abrasion sample. The reduction in abrasion was within the margin of error for having the same properties as the factory ABS-like matrix. The ZnO ABS nanocomposite did not follow a linear abrasion profile such as all other samples. There were points during the testing where substantially more or less material was removed. This means that the mixture of the two materials throughout the test pieces was not perfectly homogenous and varied based on layer depth. There could be several reasons for this phenomenon: particle settlement, clumping, and an uneven disruption of the mixture from the build plate. The $\mathrm{TiO}_{2} \mathrm{ABS}$-like nanocomposite did not follow this same path and performed far worse than the factory ABS-like resin similar to the tensile tests. Although the titanium did perform worse, it did follow a linear path with respect to time, as expected in an abrasion test. Because of this, a more uniform mixture can be inferred. This was the cause for the higher tensile stress and lower standard deviation seen compared to its $\mathrm{ZnO}$ ABS-like counterpart. The physical difference in size between the two nanoparticles was at most 20 nanometers but only about 10-15 nanometers on average. The density difference of $1.38 \mathrm{~g} / \mathrm{cm}^{-3}$ between the two nanoparticles contributed to the difference in the compatibility with the ABS-like resin. As the $\mathrm{ZnO}$ has a higher density, there would be less material added per gram than the $\mathrm{TiO}_{2}$, leading to reduced physical attributes.

Although the ABS-like material did not beneficially pair with the nanoparticles, the base resin did. The resin material saw a significant improvement in all physical properties when paired with a nanoparticle and still reattained great printing attributes. When analyzing the tensile testing data, the first noticeable difference was the increased tensile testing strength in the $\mathrm{ZnO}$ nanocomposite. When looking at the ABS-like samples, the $\mathrm{ZnO}$ nanoparticles performed worse than the $\mathrm{TiO}_{2}$, but the opposite appeared to happen here with the base resin. The $\mathrm{ZnO}$ showed a $38.4 \%$ improvement in tensile strength and a $52 \%$ decrease in strain. This result was expected as the nanocomposite created a much more rigid part that could withstand higher stresses as the nanoparticle helped disperse the load. These results reinforce the idea that a superior bond between the base resin and the nanoparticles took place compared to the ABS-like resin. $\mathrm{ZnO}$ did not outperform $\mathrm{TiO}_{2}$ 
in abrasion testing, though, as the type of degradation the part experiences will likely not increase from one nanoparticle to the next. The increase in abrasion resistance for both resin nanocomposites was not the only improved property, but also an improvement in abrasion consistency. Out of all abrasion tests performed, the resin nanocomposites demonstrated the most linear degradation curve with respect to time. This further promotes the theory that there was an improved bonding with the base resin and excellent homogeneity.

Water contact testing demonstrated that there was little effect on the surface of the materials. $\mathrm{TiO}_{2}$ was used as previous studies have shown that it tends to create hydrophobic surfaces after being exposed to the sun [? ]. As all testing was conducted indoors and long-term sun exposure was not tested, these attributes were not observed on our samples. The water absorption testing did produce results as expected for polymer-based materials and would, therefore, not be a barrier for fluid implementation. Further research into surface modification through sun exposure of $\mathrm{TiO}_{2}$ specimens would help provide more supporting evidence for fluid system involvement of parts.

Finally, SEM provided adequate proof that a sufficient dispersion was achieved with minimal clumping. As these parts were printed layer by layer, the SEM imagery showed the first and last layer of the print depending on the side it was taken. This reinforces the data that particle settlement was not an issue during the printing process. This also showed that new nanoparticles will be continuously exposed to the surface as the part wears down to keep the part's antibacterial properties active.

\section{Conclusions}

This research portrayed the discovery and possible implementation of an antibacterial nanocomposite resin material that can be implemented in standard LCD printing systems. The novel nanocomposite synthesized improved the physical and chemical properties of the base resin. All materials used were easily sourced and can be manufactured without the use of expensive machinery. In addition, the parts were easily printable on a system that allowed for more design freedom than previously seen in traditional manufacturing processes. Multiple complex parts were produced to provide evidence of different use cases. With the published research of the antibacterial effects of the nanoparticles used and the success of the trials documented within, this process could be effectively implemented in many healthcare industry sectors.

The utilization of two different nanoparticles helped demonstrate a factory resins ability to act as a matrix material across differing compounds. In addition, both nanoparticles have demonstrated antibacterial properties, so they were selected as possible reinforcements. Throughout the testing, $\mathrm{ZnO}$ was analyzed to be the best reinforcement material as it led to a more significant improvement in mechanical tests. The $\mathrm{TiO}_{2}$ nanocomposite showed a $43.5 \mathrm{MPa}$ decrease in modulus and a 4.2 MPa decrease in ultimate tensile stress compared to its $\mathrm{ZnO}$ counterpart. Using $\mathrm{TiO}_{2}$ would be beneficial if looking for improved water resistance in outdoor settings.

This new nanocomposite allows for a completely new manufacturing design philosophy as it is now compatible with many AM machines. Utilizing $\mathrm{ZnO}$ and $\mathrm{TiO}_{2}$ in the 10-30 nm range ensures a proper dispersion along with providing antibacterial traits. It was discovered that the unmodified base resin material was the best matrix for nanoparticle addition, with the $\mathrm{ZnO}$ resin providing maximum tensile strength of $33.7 \mathrm{MPa}$ and Young's Modulus of 1766.8 MPa. This nanocomposite showed improved results in tensile testing, abrasion testing, and water contact angles. In addition, it was able to be 3D printed with excellent tolerances and high reliability. These results lead to the observation that a $\mathrm{ZnO}$ resin nanocomposite at a $1 \%$ addition by weight would be the best choice for photocurable AM.

Author Contributions: Conceptualization, Y.L. and C.C.; methodology, C.B. and Y.L.; validation, C.B., C.C. and Y.L.; formal analysis, C.B.; funding acquisition, C.C. and Y.L. All authors have read and agreed to the published version of the manuscript. 
Funding: This research was funded by the Presbyterian Health Foundation (PHF), grant number 20000243.This authors also appreciate the financial support provided by the Vice President for Research and Partnerships of the University of Oklahoma.

Data Availability Statement: The data presented in this study are available on request from the corresponding author.

Conflicts of Interest: The authors declare no conflict of interest.

\section{References}

1. Shick, T.M.; Abdul Kadir, A.Z.; Ngadiman, N.H.A.; Ma'aram, A. A review of biomaterials scaffold fabrication in additive manufacturing for tissue engineering. Bioact. Compat. Polymers 2019, 34, 415-435. [CrossRef]

2. Harun, W.; Kamariah, M.; Muhamad, N.; Ghani, S.; Ahmad, F.; Mohamed, Z. A review of powder additive manufacturing processes for metallic biomaterials. Powder Technol. 2018, 327, 128-151. [CrossRef]

3. Herren, B.; Saha, M.C.; Altan, M.C.; Liu, Y. Development of ultrastretchable and skin attachable nanocomposites for human motion monitoring via embedded 3D printing. Compos. Part B Eng. 2020, 200, 108224. [CrossRef]

4. Charara, M.; Abshirini, M.; Saha, M.C.; Altan, M.C.; Liu, Y. Highly sensitive compression sensors using three-dimensional printed polydimethylsiloxane/carbon nanotube nanocomposites. J. Intell. Mater. Syst. Struct. 2019, 30, 1216-1224. [CrossRef]

5. McCullough, E.J.; Yadavalli, V.K. Surface modification of fused deposition modeling ABS to enable rapid prototyping of biomedical microdevices. J. Mater. Process. Technol. 2013, 213, 947-954. [CrossRef]

6. Wu, D.; Zhao, Z.; Zhang, Q.; Qi, H.J.; Fang, D. Mechanics of shape distortion of DLP 3D printed structures during UV post-curing. Soft Matter. 2019, 15, 6151-6159. [CrossRef] [PubMed]

7. Quan, H.; Zhang, T.; Xu, H.; Luo, S.; Nie, J.; Zhu, X. Photo-curing 3D printing technique and its challenges. Bioact. Mater. 2020, 5, 110-115. [CrossRef] [PubMed]

8. Ofosu, A. Clostridium difficile infection: A review of current and emerging therapies. Ann Gastroenterol. 2016, 29, 147. [CrossRef]

9. Choi, K.; Suh, K.; Muldoon, K.; Roth, V.; Forster, A. Hospital-acquired Clostridium difficile infection: An institutional costing analysis. Hosp. Infection. 2019, 102, 141-147. [CrossRef]

10. Hampton, T. Report reveals scope of US antibiotic resistance threat. JAMA 2013, 310, 1661-1663. [CrossRef]

11. Best, E.L.; Fawley, W.N.; Parnell, P.; Wilcox, M.H. The potential for airborne dispersal of Clostridium difficile from symptomatic patients. Clin. Infect. Dis. 2010, 50, 1450-1457. [CrossRef]

12. Best, E.; Sandoe, J.; Wilcox, M. Potential for aerosolization of Clostridium difficile after flushing toilets: The role of toilet lids in reducing environmental contamination risk. Hosp. Infection. 2012, 80, 1-5. [CrossRef] [PubMed]

13. Aithinne, K.A.; Cooper, C.W.; Lynch, R.A.; Johnson, D.L. Toilet plume aerosol generation rate and environmental contamination following bowl water inoculation with Clostridium difficile spores. Am. Infect.Control 2019, 47, 515-520. [CrossRef]

14. Jesline, A.; John, N.P.; Narayanan, P.; Vani, C.; Murugan, S. Antimicrobial activity of zinc and titanium dioxide nanoparticles against biofilm-producing methicillin-resistant Staphylococcus aureus. Appl. Nanosci. 2015, 5, 157-162. [CrossRef]

15. Fonseca, B.B.; Silva, P.L.A.P.A.; Silva, A.C.A.; Dantas, N.O.; de Paula, A.T.; Olivieri, O.C.L.; Beletti, M.E.; Rossi, D.A.; Goulart, L.R. Nanocomposite of Ag-Doped $\mathrm{ZnO}$ and $\mathrm{AgO}$ nanocrystals as a preventive measure to control biofilm formation in eggshell and salmonella spp. Entry into eggs. Front. Microbiol. 2019, 10, 217. [CrossRef] [PubMed]

16. Sezgin, E.; Keçeci, M.E.; Akmaz, S.; Koc, S.N. Heterogeneous Cr-zeolites (USY and Beta) for the conversion of glucose and cellulose to 5-hydroxymethylfurfural (HMF). Cellulose 2019, 26, 9035-9043. [CrossRef]

17. El-Megharbel, S.M.; Alsawat, M.; Al-Salmi, F.A.; Hamza, R.Z. Utilizing of (zinc oxide nano-spray) for disinfection against "SARS-CoV-2" and testing its biological effectiveness on some biochemical parameters during (COVID-19 pandemic) - “ ZnO nanoparticles have antiviral activity against (SARS-CoV-2)". Coatings 2021, 11, 388. [CrossRef]

18. Rai, P.K.; Usmani, Z.; Thakur, V.K.; Gupta, V.K.; Mishra, Y.K. Tackling COVID-19 pandemic through nanocoatings: Confront and exactitude. Curr. Res. Green Sustainable Chem. 2020, 3, 100011. [CrossRef]

19. Brunet, L.; Lyon, D.Y.; Hotze, E.M.; Alvarez, P.J.; Wiesner, M.R. Comparative photoactivity and antibacterial properties of C60 fullerenes and titanium dioxide nanoparticles. Environ. Sci. Technol. 2009, 43, 4355-4360. [CrossRef]

20. Sirelkhatim, A.; Mahmud, S.; Seeni, A.; Kaus, N.H.M.; Ann, L.C.; Bakhori, S.K.M.; Hasan, H.; Mohamad, D. Review on zinc oxide nanoparticles: Antibacterial activity and toxicity mechanism. Nano-micro Lett. 2015, 7, 219-242. [CrossRef]

21. Moritz, M.; Małgorzata, G.-M. The newest achievements in synthesis, immobilization and practical applications of antibacterial nanoparticles. Chem. Eng. J. 2013, 228, 596-613. [CrossRef]

22. Ann, L.C.; Mahmud, S.; Bakhori, S.K.M.; Sirelkhatim, A.; Mohamad, D.; Hasan, H.; Seeni, A.; Rahman, R.A. Effect of surface modification and UVA photoactivation on antibacterial bioactivity of zinc oxide powder. Appl. Surf. Sci. 2014, 292, 405-412. [CrossRef]

23. Yamamoto, O. Influence of particle size on the antibacterial activity of zinc oxide. Int. J. Inorg. Mater. 2001, 3, 643-646. [CrossRef]

24. Raghupathi, K.R.; Koodali, R.T.; Manna, A.C. Size-dependent bacterial growth inhibition and mechanism of antibacterial activity of zinc oxide nanoparticles. Langmuir 2011, 27, 4020-4028. [CrossRef] [PubMed]

25. Komissarenko, D.A.; Sokolov, P.S.; Evstigneeva, A.D.; Shmeleva, I.A.; Dosovitsky, A.E. Rheological and curing behavior of acrylate-based suspensions for the DLP 3D printing of complex zirconia parts. Materials 2018, 11, 2350. [CrossRef] 
26. Guo, J.; Zeng, Y.; Li, P.; Chen, J. Fine lattice structural titanium dioxide ceramic produced by DLP 3D printing. Ceram. Int. 2019, 45, 23007-23012. [CrossRef]

27. Cacaci, M.; Martini, C.; Guarino, C.; Torelli, R.; Bugli, F.; Sanguinetti, M. Graphene oxide coatings as tools to prevent microbial biofilm formation on medical device. Adv. Microbiol. Infectious Dis. Publ. Health 2019, 1282, 21-35.

28. Rizzello, L.; Cingolani, R.; Pompa, P.P. Nanotechnology tools for antibacterial materials. Nanomedicine 2013, 8, 807-821. [CrossRef]

29. Miyauchi, T.; Yamada, M.; Yamamoto, A.; Iwasa, F.; Suzawa, T.; Kamijo, R.; Baba, K.; Ogawa, T. The enhanced characteristics of osteoblast adhesion to photofunctionalized nanoscale TiO2 layers on biomaterials surfaces. Biomaterials 2010, 31, 3827-3839. [CrossRef]

30. Wang, J.; Weng, B.; Larson, P.; Liu, Y. Synthesis and characterization of self-assembled ZnO nanoarrays on hybrid structural fibers. Surf. Interfaces 2019, 16, 188-193. [CrossRef]

31. Zhu, W.; Ma, X.; Gou, M.; Mei, D.; Zhang, K.; Chen, S. 3D printing of functional biomaterials for tissue engineering. Curr. Opin. Biotechnol. 2016, 40, 103-112. [CrossRef] [PubMed]

32. Zhang, J.; Hu, Q.; Wang, S.; Tao, J.; Gou, M. Digital light processing based three-dimensional printing for medical applications. Int. J. Bioprint. 2020, 6. [CrossRef] [PubMed]

33. Patel, D.K.; Sakhaei, A.H.; Layani, M.; Zhang, B.; Ge, Q.; Magdassi, S. Highly stretchable and UV curable elastomers for digital light processing based 3D printing. Adv. Mater. 2017, 29, 1606000. [CrossRef] [PubMed]

34. Kuang, X.; Zhao, Z.; Chen, K.; Fang, D.; Kang, G.; Qi, H.J. High-speed 3D printing of high-performance thermosetting polymers via two-stage curing. Macromol. Rapid Commun. 2018, 39, 1700809. [CrossRef]

35. Sherman, S.L.; Kadioglu, O.; Currier, G.F.; Kierl, J.P.; Li, J. Accuracy of digital light processing printing of 3-dimensional dental models. Am. J. Orthod. Dentofacial Orthop. 2020, 157, 422-428. [CrossRef]

36. Kardar, P.; Amini, R. Self-cleaning treatment on historical stone surface via titanium dioxide nanocoatings. Pigment. Resin Technol. 2019, 48, 404-408. [CrossRef] 